\title{
Palato-gingival groove: An innocuous culprit for endo-perio lesion
}

\author{
Rafeza Sultana and Md. Shamsul Alam
}

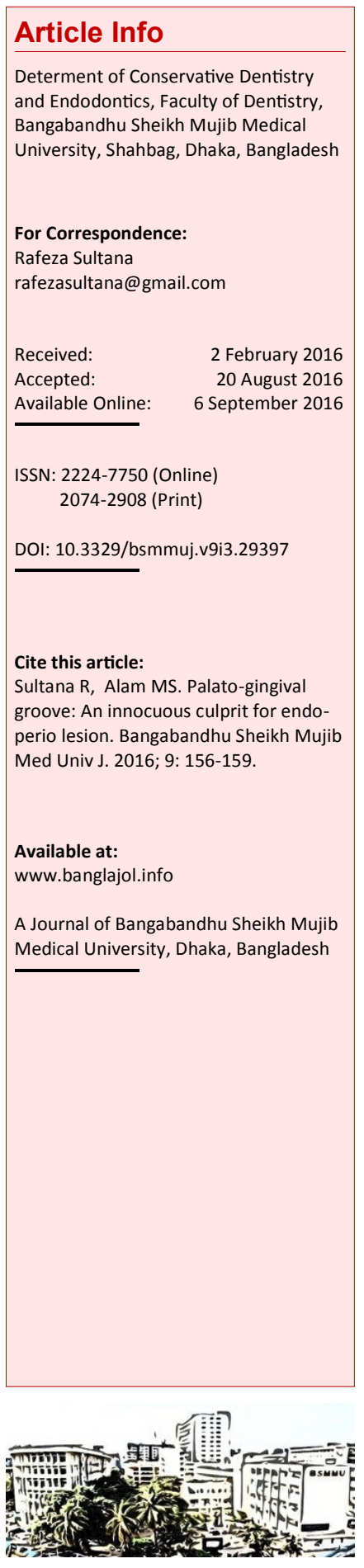

\section{Abstract}

This case report represents the clinical management of tooth with palato-gingival groove in a right maxillary lateral incisor with endo-perio lesion leading to dento-alveolar abscess and sinus tract. The right maxillary lateral incisor was examined clinico-radiographically. On clinical examination, the offending tooth revealed localized swelling and an intraoral draining sinus pointing on the labial gingiva without any evidence of caries, discoloration and trauma. The palatal surface of lateral incisor showed a groove with mild calculus embedded in it. The radiographic examination revealed periapical radiolucency. This case provides an evidence of morphological defect of tooth. Complete clinical and radiological examination and adequate knowledge of such morphological/ developmental defects of teeth are necessary for recognition and identification especially because of their diagnostic complexity and further consequences.

\section{Introduction}

Maxillary lateral incisors are located in those regions are considered to be the areas of embryological danger.1 A large number of minor and major malformations occur in this area. Such as dens in dente, peg lateral incisors, cleft palate, globule-maxillary cyst, missing or supernumerary tooth. One more anomaly occurring in that area is palato-gingival groove. It is one such morphological defect, most frequently found on the palatal surface of the maxillary lateral incisors.?

The etiology of groove formation is not fully understood. But it is thought that when the enamel organ and the Hertwing's root sheath become infolded. The palato-gingival groove is formed and has been speculated to an aborted formation of additional root. 3 The palatogingival groove has the likeness to dens invaginatus. However, both of them occurs due to an infolding of the epithelium, where the palato-gingival groove resulting in a groove and an invagination resulting in a circular opening. 4 The name of this anomaly has a wide range of diversity like the palato-gingival groove, the radicular lingual groove, the palatoradicular groove, the facial-radicular groove, and the disto-lingual groove. $\frac{5}{5}$

The palato-gingival groove appears in funnel shaped which forms a recess for bacterial plaque and calculus accumulation making it difficult in detection for the patients as well as professionals. Based on depth and extention, the palato-gingival groove can be classified in to mild, moderate and complex. Mild one terminates at the cemento-enamel junction whereas moderate grooves continue apically along the root surface. The complex one is deeply grooved defects that separate an acessory root from the main root trunk. $\underline{6}$

Many studies have demonstrated a prevalence rate for palatal groove in lateral incisors of about 2.8 to $8.5 \% .7$ The grooves on the root extended less than $5 \mathrm{~mm}$ is $43 \%$, extended between $6-10 \mathrm{~mm}$ is $47 \%$ and only $10 \%$ is in more than $10 \mathrm{~mm} . \underline{8}$

\section{Case Report}

A 30 year old female patient came to the Department of Conservative Dentistry and Endodontics, BSMMU with the complaint of occasional episodes of swelling of gum and purulent discharge in relation to upper right lateral incisor. Her medical history was noncontributory. There was no history of trauma, caries nor any discoloration of tooth. Intra-oral examination showed localized swelling and an intraoral draining sinus pointing on the labial gingiva at the apex of right lateral incisor. The palatal surface of lateral incisor showed fossa with mild calculus embedded in it (Figure 1). Periodontal examination revealed mild bleeding on probing and a narrow periodontal pocket alongside the groove. Other aspects were revealed normal. There was no mobility associated with it. To locate the origin of the sinus, a gutta percha cone (\#25) was inserted in 


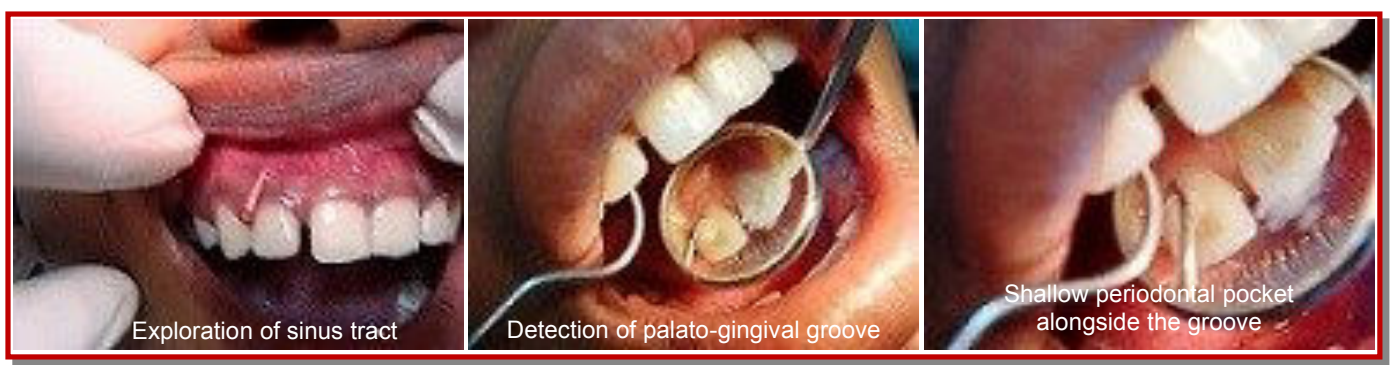

Figure 1: Palato-gingival groove

to its course. Radiograph was taken where the gutta percha cone pointed towards the apex of the offending tooth (Figure 2). Radiograph also showed periapical radiolucency (about $3 \mathrm{~mm}$ ). On vitality test the tooth was revealed non-vital. The findings were suggestive of endo-perio lesion associated with pulpal necrosis and localized periodontitis.

Therefore, the case was diagnosed as type-I endoperio lesion due to palato-gingival groove (mild type).

Treatment plan comprised of oral prophylaxis followed by non-surgical endodontic management provided three dimensional obturation and repair of palato-gingival groove with glass ionomer cement (Type II).

The consent of the patient was taken. After mouth preparation, a straight line access cavity was prepared. The necrotic pulp remnants were removed, washed out by $2.5 \% \mathrm{NaOCl}$ and normal saline. Working length measuring radiograph was taken. It was established as $20 \mathrm{~mm}$. Then the canal was prepared up to $\# 50 \mathrm{~K}$ file using standardized technique along with copious irrigation with $\mathrm{NaOCl}$ and normal saline. The canal was dried with paper point. $\mathrm{Ca}(\mathrm{OH})_{2}$ paste was placed as an interappointment dressing for 7 days. The access opening was tightly conserved with $\mathrm{ZnO}$ eugenol cement. At the following appointment the canal was found dry. Then the canal was irrigated with normal saline and dried with paper point. Before obturation, a master point was inserted into the canal and felt the tug back. Then the tooth was intervals up to 4 years.

\section{Discussion}

The patient was advised for follow-up at 12 months

Palato-gingival groove is such a developmental defect that has the tendency for the maxillary lateral incisor. As the palato-gingival groove exhibits variable extent and depth, may or may not involve a communication between pulp cavity and periodontal tissue. Initially the depth of these grooves is more after root formation and become shallow with increasing age due to deposition of dentin. The palato-gingival groove is one of the important entities and could manifest either as true endodontic lesion, periodontal disease or may appear as a combined endo-perio lesion. 9

For anatomical region, the palato-gingival groove is an ideal plaque trap for promoting periodontal breakdown and pulp necrosis. The existence of communication between the pulp chamber and the periodontium may be the reasons for the occurance of combined endo-perio lesion. Friedman and Goultschin have suggested pulpal necrosis followed by apical periodontitis is often earliest manifestation of palato-gingival groove. $\underline{10}$

For the treatment of palato-gingival groove, clinician should be aware of its incidence and method. Seldom the palato-gingival groove can be seen on radiographic examination in the form of a

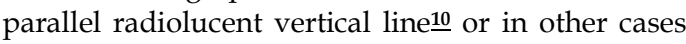

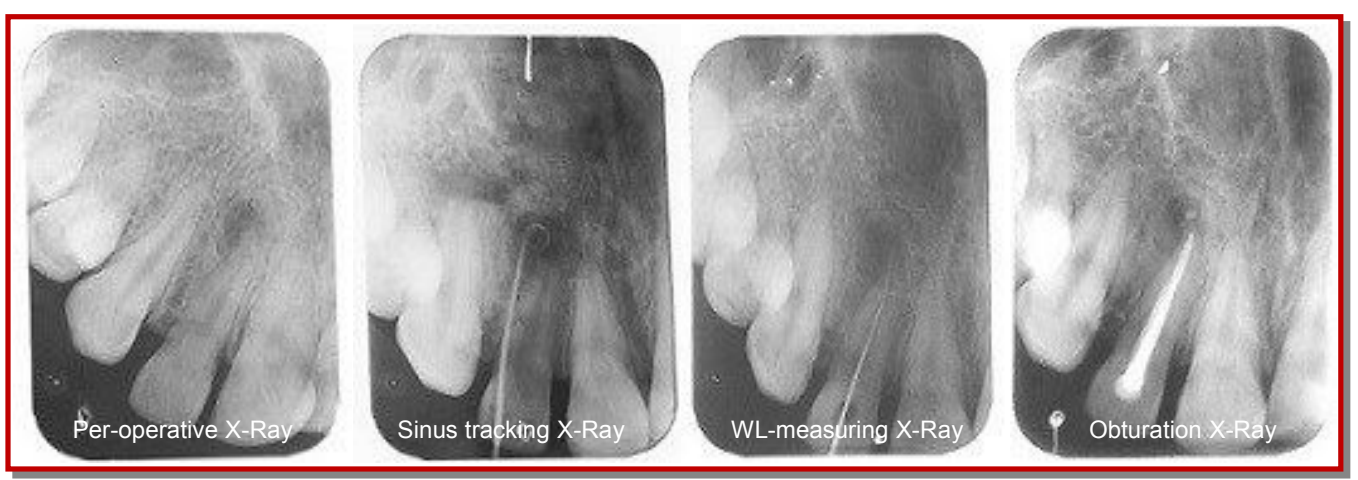
not following the root canal. $\underline{11}$

As palato-gingival groove may result in radicular and pulpual pathosis, it requires early diagnosis and treatment. This crevice like channel is a locus of plaque and calculus accumulation, which acts as a secondary local etiologic factor for encouraging the $\mathrm{d}$ e velopme $\mathrm{nt}$ of periodontitis. A patient with

obturated with ZOE sealer lateral condensation technique and final restoration was made with glass ionooperative radiograph was taken at the same visit for palato-gingival groove was repaired with glass . 
palato-gingival groove may show no symptoms at all or have the symptoms of a periodontal or acute dento-alveolar abscess. Commonly a lesion related to a groove is characterized by recurrent symptomatic episodes. $\underline{\mathbf{1 2}}$

The pulp which is situated in the radicular groove is also affected by bacteria. The accessory foramen and lateral canals situated along the floor or side walls of the groove by which bacteria and their products may enter in to the pulp. One more route of the bacterial invasion into the pulp is via the exposed dentinal tubules on the side of the groove where surface resorption may occur as an inflammatory response. $\underline{13}$

There is a variation of treatment from case to case. For preventive measure, early diagnosis of the case is very crucial. Teeth with deep palatal groove should be treated with fissure sealant before plaque and food impaction and breakdown of the periodontal structures. $\underline{14}$

For the treatment of this condition, several modalities have been suggested. There is general agreement that they have predictable failures. Various treatment options have been recommended such as conventional root canal treatment; combined root canal therapy followed by saucerization of the defect with flowable composite, intentional replantation and guided tissue regeneration according to severity. $\underline{\underline{15}}$

In the most recent decades, the guided tissue regeneration, mechanical barrier have been used to halt the epithelium down growth along the root surface, allowing periodontal ligament, cementum and bone to regenerate along periodontally diseased roots. Various therapeutics such as calcium sulphate, collagen methyl cellulose acetate, enamel matrix protein etc have used as mechanical barrier to allow periodontal regeneration. 16

The prognosis of a tooth with palato-gingival groove mainly depends on location of the groove, severity of the periodontal problem, accessibility of the defect and the type of groove either shallow or deep, long or short.17

In this case report, patient presented with true endodontic involvement (type I endo-perio lesion), as an earlier manifestation of palato-gingival groove. So, non-surgical endodontic treatment was done provided three dimensional obturation. The groove was repaired with glass ionomer cement and advised for periodic follow-up.

For the repairment of groove, glass ionomer cement was used because of its antibacterial activity and the property of chemical adhesion to the tooth structure. Clinical and histological studies have been shown that there is an apical and CT adherence to the glass ionomer cement during the healing process. 18

\section{Conclusion}

Palato-gingival groove is an enigma and it is considered as a silent killer that can pose dilemmas for diagnosis and clinical management. Therefore, the early detection of clinical signs and appropriate diagnostic tests are of paramount important to prevent incorrect diagnosis and treatment.

\section{References}

1. Anderegg CR, Meitzer DG. Treatment of the palato-gingival groove with guided tissue regeneration: Report of 10 cases. J Periodontol. 1993; 64: 72-74

2. Andreana SA. Combined approach of treatment for developmental groove associated periodontal defect: A case report. J Periodontal. 1998; 69: 60107.

3. August DS. The radicular ligual groove: An overlooked differential diagnosis. J Am Dent Assoc. 1978; 90: 1037-39.

4. Bacic M, Karakas Z, Kaic Z, Sutalo J. The association between palatal grooves in upper incisors and periodontal complications. J Periodontal. 1990; 61: 197-99.

5. Ceciflia MS, Lara VS, Moraes IG. The palatogingival groove a cause of failure in root canal treatment. Oral Surg Oral Med Oral Pathol Oral Radiol Endod. 1998; 85: 94-98.

6. Rachana D, Nadig P, Nadig G. The palata groove: Application of computed tomography in its detection: A case report. J Conserv Dent. 2007; 10: 83-88.

7. Everatt FG, Kermer GM. The disto-lingual groove in the maxillary lateral incisor. J Periodontol. 1972; 43: 352-61.

8. Goon WW, Carpenter WM, Braces NM, Ahlfeld RJ. Complex facial radicular groove in a maxillary lateral incisor. J Endod. 1991; 17: 244-48.

9. Pecora JD, Damia M, Sousaneto O. In vitro study of redicular grooves in maxillary incisors. Braz Dent J. 1991; 2: 69-73.

10. Kogan S. The prevalence, location and conformation of palato radicular grooves in maxillary incisors. J Peridontol. 1986; 57: 231.

11. Kozlovsky A, Tal H, Yechezkiely N, Mozes O. Facial radicular grooves in a maxillary central incisor: A case report. J Periodontol. 1988; 59: 61517.

12. Lee RW, Lee EC, Poon RY. Palato gingival grooves in maxillary incisors: A possible 
predisposing factoto localized periodontal disease. Brit dent J. 1968; 2: 14-18.

13. Hulsmann M. Dens invaginatus: Aetiology, classification, prevalence, diagnosis and treatment considerations. Int Endod J. 1997; 30: 79-90.

14. Hans MK, Srinivus RS, Shetty SB. Management of lateral incisor with palatal redicular groove. Indian J Dent Res. 2010; 21: 306-08.

15. Kerezoudis NP, Siskos GJ, Tsatsas V. Bilateral buccal radicular groove in maxillary incisors: Case report. Int Endo J. 2003; 36: 898-906.
16. Robinson SF, Cooley RI. Palato-gingival groove lesions: Recognition and treatment. Gen Dent. 1988; 36: 340-42.

17. Schafer E, Cankay R, Ott K. Malformations in maxillary incisors: Case report of radicular palatal groove. Endod Dent Traumatol. 2000; 16: 132-37.

18. Gound TG, Mze GI. Treatment options for the redicular lingual groove: A review and discussion. Pract Periodont Aesthetic Dent. 1998; 10: 369-75. 